\title{
A Numerical Method for Analyzing Electromagnetic Scattering Properties of a Moving Conducting Object
}

\author{
Lei Kuang, Shouzheng Zhu, Jianjun Gao, Zhengqi Zheng, and Danan Dong \\ School of Information Science and Technology, East China Normal University, Shanghai 200241, China \\ Correspondence should be addressed to Lei Kuang; lkuang@ee.ecnu.edu.cn
}

Received 19 October 2013; Revised 1 January 2014; Accepted 15 January 2014; Published 23 February 2014

Academic Editor: Gaobiao Xiao

Copyright (C) 2014 Lei Kuang et al. This is an open access article distributed under the Creative Commons Attribution License, which permits unrestricted use, distribution, and reproduction in any medium, provided the original work is properly cited.

\begin{abstract}
A novel numerical approach is developed to analyze electromagnetic scattering properties of a moving conducting object based on the finite-difference time-domain (FDTD) algorithm. Relativistic boundary conditions are implemented into the FDTD algorithm to calculate the electromagnetic field on the moving boundary. An improved technique is proposed to solve the scattered field in order to improve the computational efficiency and stability of solutions. The time-harmonic scattered field from a one-dimensional moving conducting surface is first simulated by the proposed approach. Numerical results show that the amplitude and frequency of the scattered field suffer a modulation shift. Then the transient scattered field is calculated, and broadband electromagnetic scattering properties of the moving conducting surface are obtained by the fast Fourier transform (FFT). Finally, the scattered field from a two-dimensional moving square cylinder is analyzed. The numerical results demonstrate the Doppler effect of a moving conducting object. The simulated results agree well with analytical results.
\end{abstract}

\section{Introduction}

With the development of aerospace technology and deep space exploration, the analysis of electromagnetic scattering properties of moving bodies with a high speed has gained more and more attention recently. It has wide applications in the radar detection of high-speed moving bodies.

The existing analytical theory of the electromagnetic scattering from moving bodies can be carried out for regular shapes. Strauss [1] obtained the scattering operator in the presence of a moving object. The electromagnetic jump conditions were introduced at a moving boundary by Costen and Adamson [2]. Using the Lorentz transformation, Michielsen et al. [3] developed the electromagnetic waves scattered by a uniform moving obstacle. Arnaoudov and Venkov [4] developed an analytical model to analyze the low-frequency scattering process generated by a plane electromagnetic field that is incident upon a moving perfectly conducting. Georgiev [5] studied the inverse electromagnetic scattering problem for moving surfaces. However, it is difficult to analyze general two- or three-dimensional bodies, since closed-form solutions cannot be obtained when the body shape, composition, and transition are arbitrary.

A numerical approach is required to solve the electromagnetic field around the moving body with irregular shapes. Ho [6] simulated the scattered field from a rotating twodimensional cylinder using passing center swing back grids technique. Based on the FDTD algorithm [7-9], Sahrani and Kuroda [10] analyzed the electromagnetic field with moving boundaries by combining the Lorentz transformation. They did not, however, discuss how to introduce the incident plane wave source into the FDTD computational domain to deal with the scattered field from moving bodies. Subsequently a new Lorentz-FDTD algorithm is proposed by Zheng et al. [11]. They discussed how to introduce the incident plane wave in the moving reference system in the calculations. However, the Lorentz transformation [12] requires at least two frames. And a system transformation is needed; that increases the calculation complexity. Harfoush et al. [13] calculated electromagnetic scattering from the conducting surfaces by the FDTD algorithm. However, it cannot be extended to three-dimensional electromagnetic scattering, 
since the calculation of the electromagnetic field at the moving interface is based on the reflection model of an infinite conducting plane surface.

Based on the FDTD method, we calculated the electromagnetic field on the moving boundary with relativistic boundary conditions. We analyzed harmonic responses and transient responses of a one-dimensional moving conducting surface. We also analyzed the scattering properties of a two-dimensional moving conducting square cylinder. The proposed numerical approach uses no system transformation and gives the solution directly in the time domain. The Doppler effect of the scattered field from a moving conducting surface was observed in the simulation. Although scattering properties of one- and two-dimensional moving objects were discussed in the paper, the proposed numerical approach can be extended to the three-dimensional case. This will be our further research.

The present paper is organized as follows. Section 2 introduces the relativistic boundary conditions and equations for calculating the electromagnetic field on a moving boundary. Section 3 briefly summarizes the FDTD method and describes the implementation of relativistic boundary conditions. Section 4 presents the analytical formula of a moving conducting surface. Section 5 discusses the simulated results of a one-dimensional moving conducting surface and a two-dimensional moving conducting square cylinder.

\section{Relativistic Boundary Conditions for a Moving Conducting Object}

For a stationary boundary separating regions 1 and 2, the tangential components of $\vec{E}$ and the normal components of $\vec{B}$ are continuous across the boundary. On the moving boundaries between region 1 and region 2, however, the components of electric field and magnetic field should satisfy relativistic boundary conditions. The derivation of these conditions, in its general form, is well presented in [14] and yields

$$
\begin{gathered}
\widehat{n} \times\left(\vec{E}_{1}-\vec{E}_{2}\right)-(\widehat{n} \cdot \vec{v})\left(\vec{B}_{1}-\vec{B}_{2}\right)=0, \\
\widehat{n} \cdot\left(\vec{D}_{1}-\vec{D}_{2}\right)=\rho_{s}, \\
\widehat{n} \times\left(\vec{H}_{1}-\vec{H}_{2}\right)+(\widehat{n} \cdot \vec{v})\left(\vec{D}_{1}-\vec{D}_{2}\right)=\vec{J}_{s}, \\
\widehat{n} \cdot\left(\vec{B}_{1}-\vec{B}_{2}\right)=0,
\end{gathered}
$$

where subscripts 1 and 2 denote fields in regions 1 and $2 ; \vec{E}, \vec{D}$, $\vec{H}$, and $\vec{B}$ are the electric field, electric flux density, magnetic field, and magnetic flux density, respectively; $\rho_{s}$ and $J_{s}$ denote the surface-charge and current densities; $\vec{v}$ is the velocity of the moving interface (assumed to be uniform). We define the surface normal $\widehat{n}$ point from region 2 to region 1 . When $\vec{v}=$ 0 or $\widehat{n} \cdot \vec{v}=0$, (la)-(1d) reduce to equations of boundaries conditions of stationary boundaries.

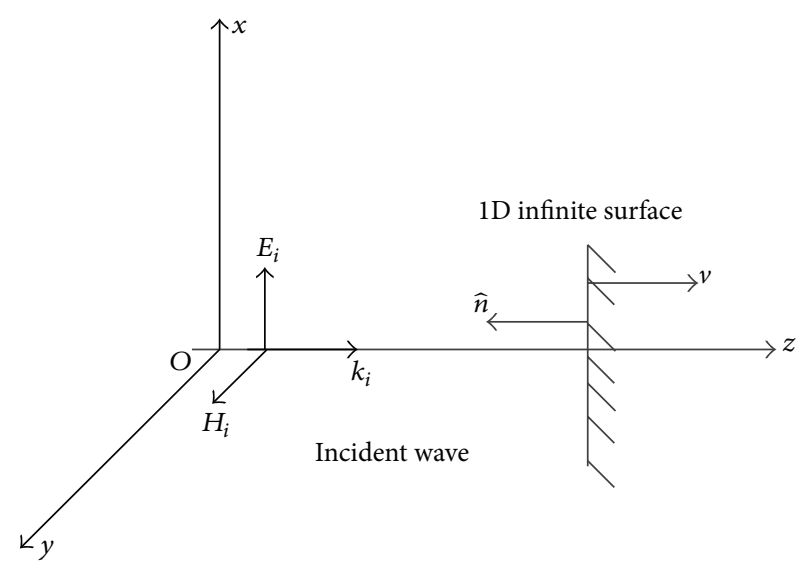

FIGURE 1: A plane wave normally incident on a moving conducting plane surface.

When we consider region 2 as a perfectly conducting medium, relativistic boundary conditions become

$$
\begin{gathered}
\widehat{n} \times \vec{E}_{1}-(\widehat{n} \cdot \vec{v}) \vec{B}_{1}=0, \\
\widehat{n} \cdot \vec{D}_{1}=\rho_{s}, \\
\widehat{n} \times \vec{H}_{1}+(\widehat{n} \cdot \vec{v}) \vec{D}_{1}=\vec{J}_{s}, \\
\widehat{n} \cdot \vec{B}_{1}=0 .
\end{gathered}
$$

A one-dimensional moving conducting surface illuminated by a plane wave is shown in Figure 1. The incident electric field is polarized in the $x$-direction and incident magnetic field is polarized in the $y$-direction. The conducting surface is parallel to the $x o y$-plane and moves along the $+z$-axis with the speed of $v$.

According to the relativistic boundary conditions, total field (including incident field and scattered field) components of $\vec{E}$ and $\vec{H}$ on the one-dimensional moving interface are given by

$$
E_{x}=v \cdot \mu \cdot H_{y}
$$

The relativistic boundary conditions can be easily implemented for the two- and three-dimensional cases. It is important to note from (2a), (2b), (2c), and (2d) that a scatterer motion perpendicular to the surface normal results in boundary conditions similar to those of a fixed object. The reason is that the term $\widehat{n} \cdot \vec{v}$ is now equal to 0 .

\section{A Numerical Model for a Moving Conducting Object}

The Finite-difference time-domain (FDTD) algorithm was introduced by Yee [7] to numerically solve Maxwell's curl equations using finite differences. In a one-dimensional case, 
Maxwell's curl equations are equivalent to the following scalar equations:

$$
\begin{gathered}
-\frac{\partial H_{y}}{\partial z}=\varepsilon \frac{\partial E_{x}}{\partial t}+\sigma E_{x} \\
\frac{\partial E_{x}}{\partial z}=-\mu \frac{\partial H_{y}}{\partial t}-\sigma_{m} H_{y}
\end{gathered}
$$

where $\sigma$ and $\sigma_{m}$ denote conductivity and magnetic inductivity, respectively.

With the FDTD algorithm, the continuous electromagnetic field in finite volume is sampled at discrete points in a space lattice and in time step. Therefore, the finite difference equation for $(4 \mathrm{a})$ is as follows:

$$
\begin{aligned}
E_{x}^{n+1}(k)= & C A(m) \cdot E_{x}^{n}(k)-C B(m) \\
& \cdot \frac{H_{y}^{n+1 / 2}(k+1 / 2)-H_{y}^{n+1 / 2}(k-1 / 2)}{\Delta z},
\end{aligned}
$$

where $C A(m)$ and $C B(m)$ are defined as

$$
\begin{aligned}
& C A(m)=\frac{1-\sigma(m) \Delta t / 2 \varepsilon(m)}{1+\sigma(m) \Delta t / 2 \varepsilon(m)}, \\
& C B(m)=\frac{\Delta t / \varepsilon(m)}{1+\sigma(m) \Delta t / 2 \varepsilon(m)},
\end{aligned}
$$

with $m=k$. Similarly, the finite difference equation for (4b) becomes

$$
\begin{aligned}
H_{y}^{n+1 / 2}\left(k+\frac{1}{2}\right)= & C P(m) \cdot H_{y}^{n-1 / 2}\left(k+\frac{1}{2}\right)-C Q(m) \\
& \cdot \frac{E_{x}^{n}(k+1)-E_{x}^{n}(k)}{\Delta z},
\end{aligned}
$$

where $C P(m)$ and $C Q(m)$ are written as

$$
\begin{aligned}
& C P(m)=\frac{1-\sigma_{m}(m) \Delta t / 2 \mu(m)}{1+\sigma_{m}(m) \Delta t / 2 \mu(m)}, \\
& C Q(m)=\frac{\Delta t / \mu(m)}{1+\sigma_{m}(m) \Delta t / 2 \mu(m)},
\end{aligned}
$$

with $m=k+1 / 2$. In these equations, superscript $n$ is the time step index and $i, j$, and $k$ are the spatial indices corresponding to the $x-, y$-, and $z$-axis, respectively. The finite difference equations for the two- and three-dimensional cases can be acquired from [7].

In the study of electromagnetic scattering from the moving conducting surface, the relativistic boundary conditions are implemented into the FDTD method. The FDTD scattering model for the moving conducting surface is shown in Figure 2. Radiation boundary conditions [15] are implemented to truncate the FDTD computational domain. The FDTD computational domain is separated into two parts. One part is the total field region including the moving conducting surface. The other part is the remaining region called the scattered field region. The incident plane wave is introduced to the total field region by the connecting

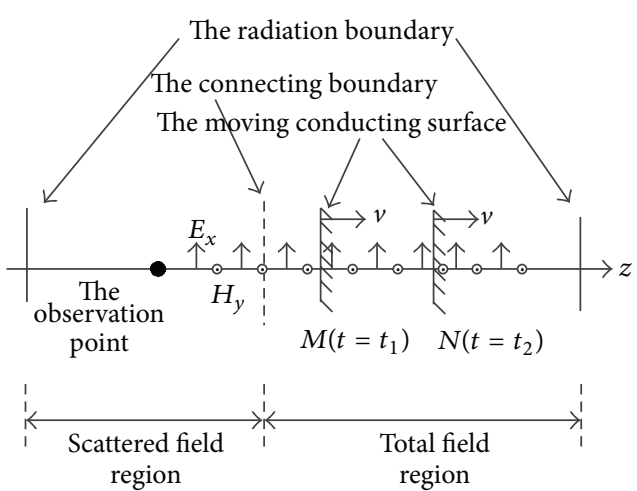

FIGURE 2: The FDTD model for the conducting surface moving.

boundary. In order to study the scattered field from the moving conducting surface, the observation point is placed in the scattered field region, shown in Figure 2.

The relativistic boundary condition is only implemented for the electric field at a moving conducting surface. According to [13], it should be enough to model the scattering from a moving object. Here, (3) should be implemented for the electric field on the moving boundary at each time step. When the surface moves, however, the moving boundary cannot be just at the position of the $E_{x}$ component in the FDTD model. At the time of $t_{1}$, the conducting surface is at the position $M$, as shown in Figure 2. The distance between the electric field component $E_{x}$ closest to the moving boundary and the moving boundary is more than $\Delta z / 2$. Subsequently, the conducting surface moves at the position $\mathrm{N}$ when $t$ is $t_{2}$. The distance between the electric field component $E_{x}$ closest to the moving boundary and the moving boundary is less than $\Delta z / 2$. Therefore, (3) is implemented only for the $E_{x}$ component closest to the moving boundary. The closest distance between the $E_{x}$ component and the moving boundary ranges from 0 to one spacial increment. The accumulated errors make the FDTD equations nonconvergent after many time step calculations.

To resolve this problem, an improved technique is proposed in the paper. From Figures 3(a) and 3(b), an arrow represents the electric component $E_{x}$ and a dot represents the magnetic component $H_{y}$. The moving conducting surface is assumed to be relatively static in the FDTD domain, as shown in Figures 3(a) and 3(b). Thus the position of the $E_{x}$ component becomes fixed at the moving boundary all the time. By introducing proper incident field values to the FDTD total field region and moving the observation point, the scattered field from the moving conducting surface can be simulated. The stability of solutions is satisfactory. Electromagnetic field components at the truncated boundaries are calculated by the radiation boundary conditions. And the rest of electromagnetic field components in the FDTD domain can be calculated by (5) and (7).

In Figure 3(c), the connecting boundary is at the position $A$ when $t=t_{1}$. Therefore, at the time of $t_{1}$, the value of the incident field at the position $A$ is introduced to excite the conducting surface being relatively static in the FDTD model, 


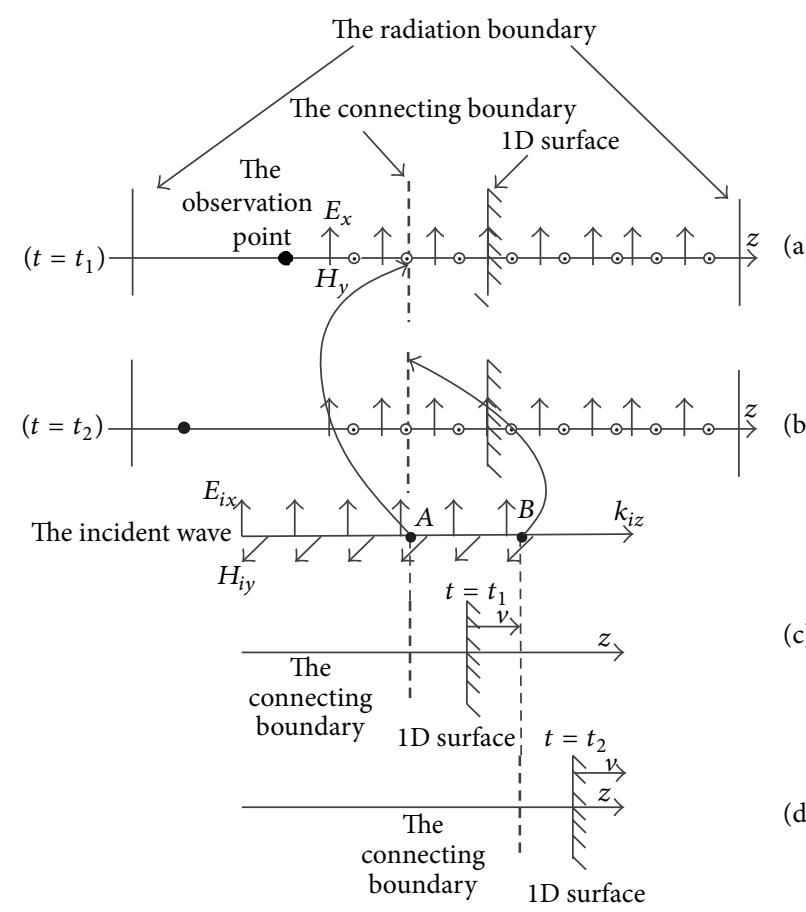

FIGURE 3: An improved FDTD model for the conducting surface being relatively static in the computational domain.

as shown in Figure 3(a). The desired value of the incident field is interpolated using the closest two points in the incident field grids. From Figure 3(d), when $t=t_{2}$, the connecting boundary moves at the position $B$. Consequently, at the time of $t_{2}$, the value of the incident field at the position $B$ should be introduced to the total field region, as shown in Figure 3(b). Since the conducting surface is assumed static in the FDTD domain, the observation point should be moved opposite to the moving direction of the conducting surface with the speed of $v$ at each time step in order to simulate relative motion between observation point and the conducting surface.

To ensure the stability of the FDTD method for a onedimensional static object, a time increment $\Delta t$ and a spatial increment $\Delta$ usually must be $\Delta t \leq \Delta / c$ and $\Delta \leq \lambda / 10$, respectively. Considering a large moving velocity, however, the mesh size should be further reduced to ensure the stability. Here, a spatial increment of $\Delta=\lambda / 20$ can guarantee the stability when the velocity is less than $0.1 c$ ( $c$ is the speed of light). A spatial increment of $\Delta=\lambda / 60$ guarantees the stability for $v>0.1 c$.

Compared with the existing methods, computation efficiency is improved by our proposed method. The Lorentz transformation method can be used to solve the scattered field from a moving object. However, both the Lorentz transformation and the inverse Lorentz transformation are needed before the final result is provided. By implementing the relativistic boundary conditions at a moving boundary, a straightforward solution is possible without any transformation in this paper. That improves the computation efficiency. Reference [13] also introduced the relativistic boundary conditions to solve the scattered field from a moving object.
However, the object is moving in the total field region with the time marching in the FDTD algorithm. Thus the total field region should include both the space occupied by the object and the moving space of the moving object. Also, the moving object should be remodeled at the updated position at each time step in the FDTD total field region. In our method, however, the moving object is assumed to be relatively static in the FDTD total region. The FDTD total field region includes the space occupied by the static object only. And the object does not need to be remodeled at each time step in the calculation. Therefore, our proposed approach reduces the computational time and storage.

The relativistic boundary conditions based on a reflection model of an infinite surface are applied in [13]. That cannot be extended to compute the scattered field from a threedimensional moving object. The relativistic boundary conditions implemented in our method can be easily extended to a three-dimensional case.

\section{Analytical Formula for the Scattered Fields from a Moving Conducting Surface}

When an incident sinusoidal plane wave of angular frequency $\omega$ and unit amplitude is normally incident on a moving infinite conducting surface with a speed $v$, the scattered electric field is given by [16]

$$
\begin{aligned}
E_{s}= & -E_{0}\left[\frac{1-v / c}{1+v / c}\right] \\
& \times \exp j\left[\left(\frac{1-v / c}{1+v / c}\right)(\omega t-k z)+2 j k\left(\frac{r_{0}-v t_{0}}{1-v / c}\right)\right],
\end{aligned}
$$

where $z_{0}=v\left(t-t_{0}\right)+r_{0}$ is the position of the surface boundary with respect to a reference point and $r_{0}$ and $t_{0}$ are initial values. It has been seen from (9) that a Doppler effect [17] is apparent in the scattered field. Both frequency and amplitude of the scattered field are changed by multiplying the same factor which is defined as

$$
\alpha=\frac{1-v / c}{1+v / c} .
$$

\section{Numerical Results}

In order to validate the accuracy of our numerical method and to illustrate the Doppler effect of a moving conducting object, numerical results are presented in this section. The solution procedure to gain the electromagnetic scattering properties of a moving conducting object mainly includes two steps. The first step is to calculate the time-domain scattered field. The second step is to obtain the frequency spectrum of the scattered field by using the fast Fourier transform (FFT).

A one-dimensional conducting plane surface is placed in the FDTD domain, as shown in Figures 3(a) and 3(b). It moves with speed of $\hat{v}=v \widehat{z}$. A positive speed of $v$ means that the surface is receding from the incident wave and a negative 


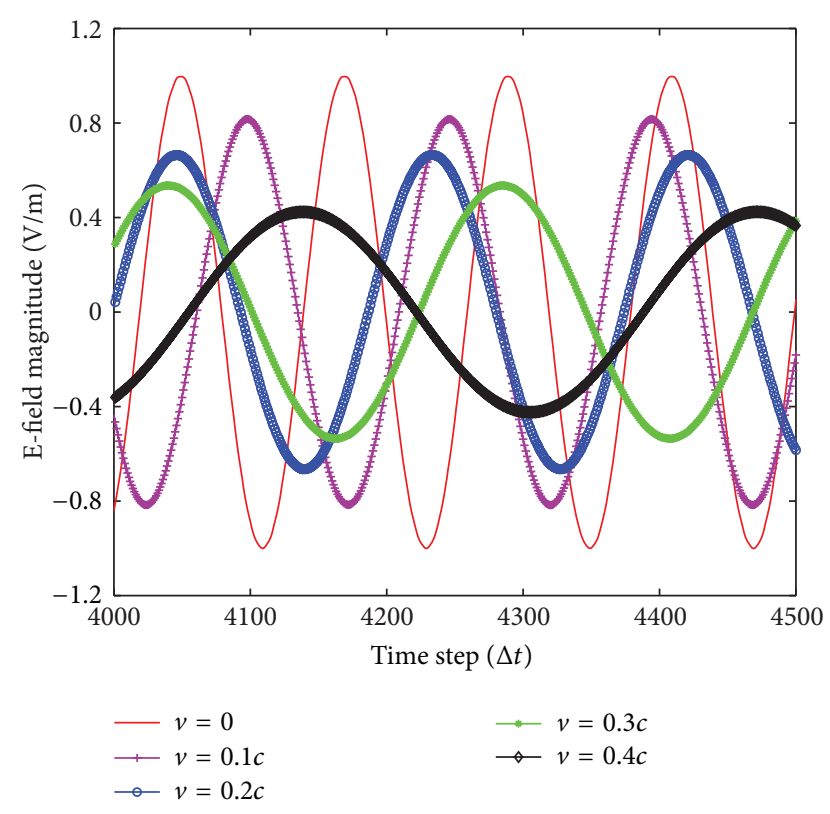

FIGURE 4: Time-domain scattered waveforms of the conducting surface moving along the $+z$-axis.

speed means that the surface is moving toward the incident wave. A sine plane wave of the frequency $f=1.0 \mathrm{GHz}$ and unit amplitude is adopted as an incident wave, which spreads along the $z$-axis normal incidence to the conducting surface. We assume that the conducting plane surface moves linearly along the $z$-axis with speeds of $v=0,0.1 c, 0.2 c, 0.3 c$, and $0.4 c \mathrm{~m} / \mathrm{s}$ ( $c$ is the speed of light in free space), respectively. In the FDTD model, the spacial increment is: $\Delta z=0.005 \mathrm{~m}$ and the time increment $\Delta t$ is determined by the Courant condition [9], which is set to be 8.3333 picoseconds. The simulated time-domain scattered waveforms and frequency spectrums of the conducting surface with different speeds are plotted in Figures 4 and 5, respectively. As seen from Figures 4 and 5, the amplitude and frequency of the scattered field are equal to those of the incident field when the conducting surface is stationary. It can be seen from Figure 4 that amplitudes of the scattered field are decreased to $0.8179,0.6663,0.5379$, and $0.4281 \mathrm{~V} / \mathrm{m}$, respectively, when the conducting surface moves with speeds of $v=0.1 c, 0.2 c, 0.3 c$, and $0.4 c \mathrm{~m} / \mathrm{s}$, respectively. And frequencies of the scattered field from the conducting surface with different speeds can be obtained by extracting peak point values of curves in Figure 5. It is obvious that frequencies of the scattered field from the conducting surface with speeds of $v=0.1 c, 0.2 c, 0.3 c$, and $0.4 c \mathrm{~m} / \mathrm{s}$ shift to 0.8178 , $0.6662,0.5378$, and $0.4279 \mathrm{GHz}$.

According to (9), when a one-dimensional moving conducting surface is excited by a plane wave, the amplitude and frequency ratio of the scattered and incident field $\alpha$ is defined as (10). Substituting the speed of $v=0,0.1 c, 0.2 c, 0.3 c$, and $0.4 \mathrm{c} \mathrm{m} / \mathrm{s}$ into (10), the theoretical results of amplitudes of scattered waves are 1.0, $0.8182,0.6667,0.5385$, and $0.4286 \mathrm{~V} / \mathrm{m}$ and those of frequencies of scattered waves are 1.0, 0.8182, $0.6667,0.5385$, and $0.4286 \mathrm{GHz}$, respectively. By comparing

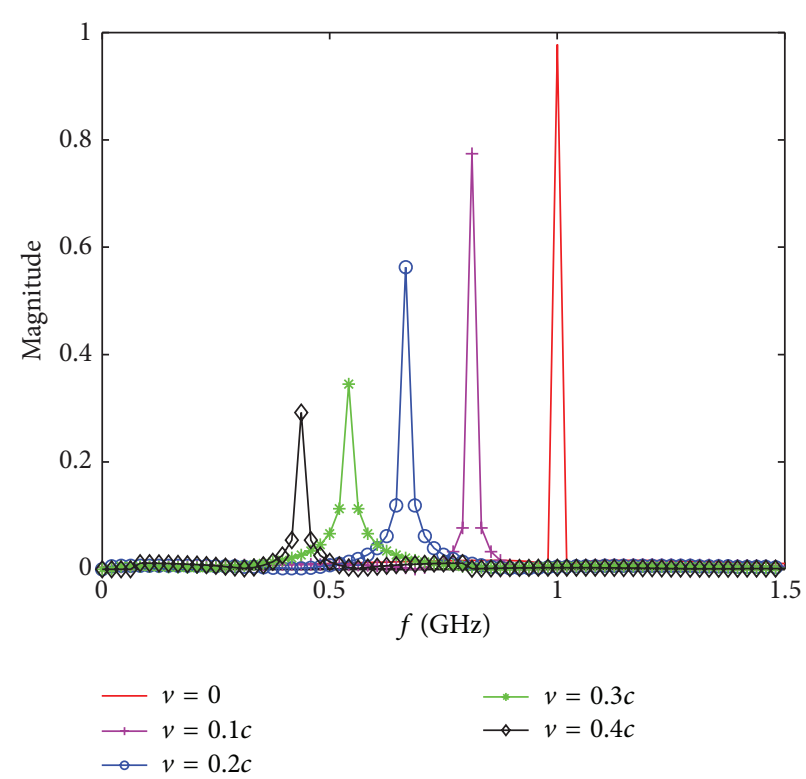

Figure 5: Frequencies of the scattered waves from the conducting surface moving along the $+z$-axis.

the theoretical results with the simulated results, a good agreement has been reached.

Assume that the speed of the conducting surface is, respectively, equal to four cases as $-0.1 c,-0.2 c,-0.3 c$, and $-0.4 c \mathrm{~m} / \mathrm{s}$. The time-domain scattered waveforms are shown in Figure 6. The simulated amplitudes of scattered waves are equal to $1.2224,1.5006,1.8581$, and $2.3345 \mathrm{~V} / \mathrm{m}$, respectively. The frequencies of scattered waves are plotted in Figure 7. By extracting peak point values of curves in Figure 7, simulated frequencies of scattered waves are equal to 1.2226, 1.5008, 1.8585 , and $2.3349 \mathrm{GHz}$, respectively.

According to (10), theoretical amplitudes of scattered waves are $1.2222,1.5000,1.8571$, and $2.3333 \mathrm{~V} / \mathrm{m}$, respectively, when the conducting surface moves with speeds of $v=-0.1 c$, $-0.2 c,-0.3 c$, and $-0.4 c \mathrm{~m} / \mathrm{s}$. And theoretical frequencies of scattered waves are 1.2222, 1.5000, 1.8571, and $2.3333 \mathrm{GHz}$, respectively. Comparison shows that the amplitudes and frequencies of scattered waves calculated by the proposed numerical method agree well with the theoretical results.

For the sake of obtaining the transient scattering of the moving conducting surface, two kinds of transient excitation sources are used in the FDTD model. A Gaussian pulse with maximum electric field amplitude of $1 \mathrm{~V} / \mathrm{m}$ is first adopted to excite the moving conducting surface, which has the following form:

$$
E_{i}(n \Delta t)=\exp \left(-\frac{4 \pi\left(n \Delta t-t_{0}\right)^{2}}{\tau^{2}}\right)
$$

where $\Delta t$ is the time increment in the FDTD simulation, $\tau$ is the pulse half-duration at the $1 / e$ point, and it determines the Gaussian width. The peak point value of the Gaussian pulse appears at $n \Delta t=t_{0}$. Here, $\tau$ and $t_{0}$ of the Gaussian pulse are set to $400 \Delta t$ and $t_{0}=750 \Delta t$. The dashed line is the incident waveform in Figure 8. 


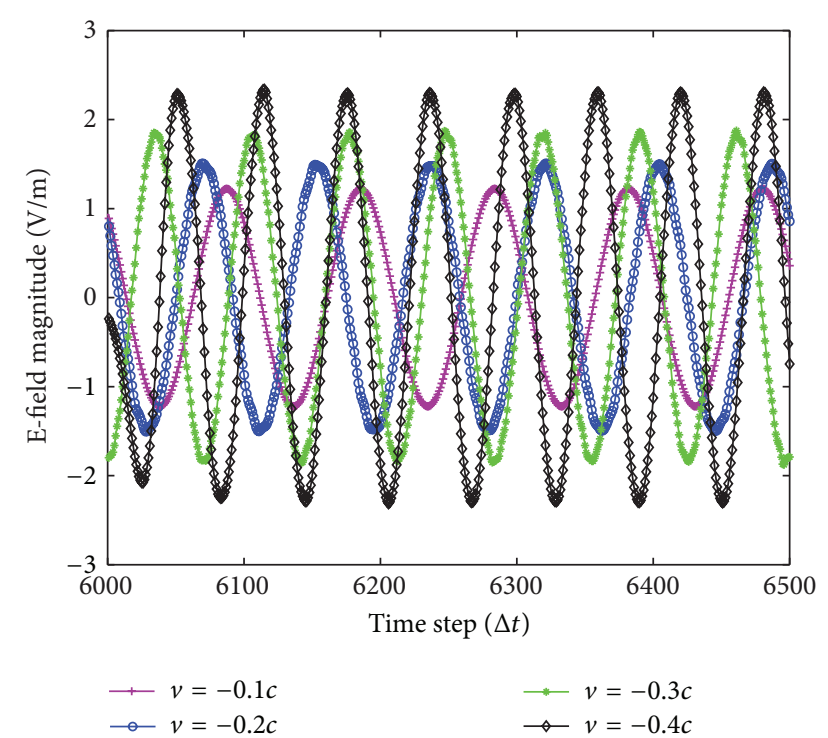

FIGURE 6: Time-domain scattered waveforms of the conducting surface moving along the $-z$-axis.

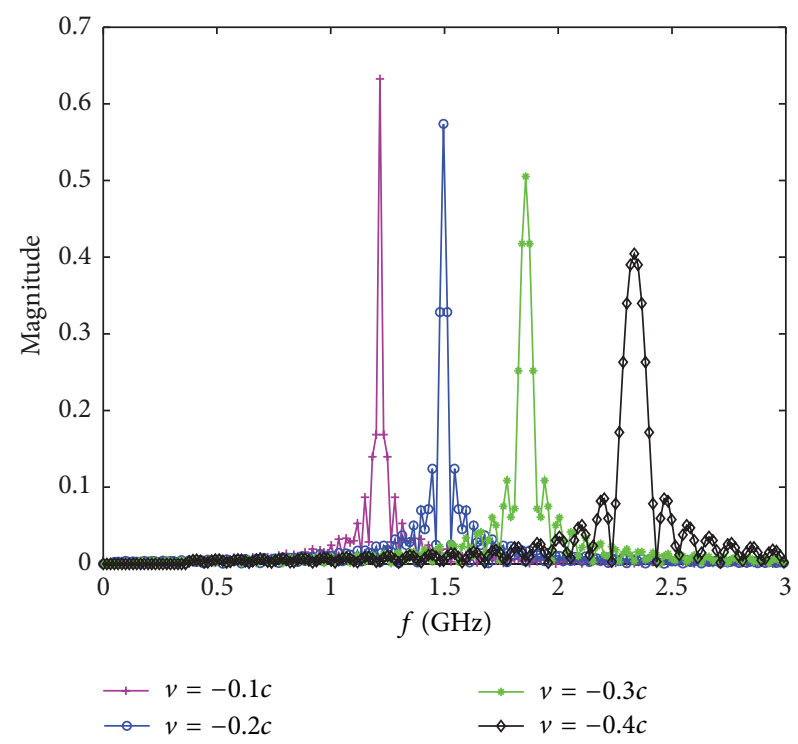

FIGURE 7: Frequencies of the scattered waves from the conducting surface moving along the $-z$-axis.

In the simulation, the speed of the moving conducting surface takes five cases of $0,0.1 c, 0.2 c, 0.3 c$, and $0.4 c \mathrm{~m} / \mathrm{s}$. Time-domain scattered waveforms of the conducting surface with different speeds are plotted in Figure 8. From Figure 8, the pulse-width of the scattered wave is broadened with the increasing moving speed. The amplitudes of simulated scattered pulses are equal to $1.0,0.8181,0.6665,0.5381$, and $0.4282 \mathrm{~V} / \mathrm{m}$, respectively. The amplitudes of simulated scattered waves show good agreement with the theoretical results.

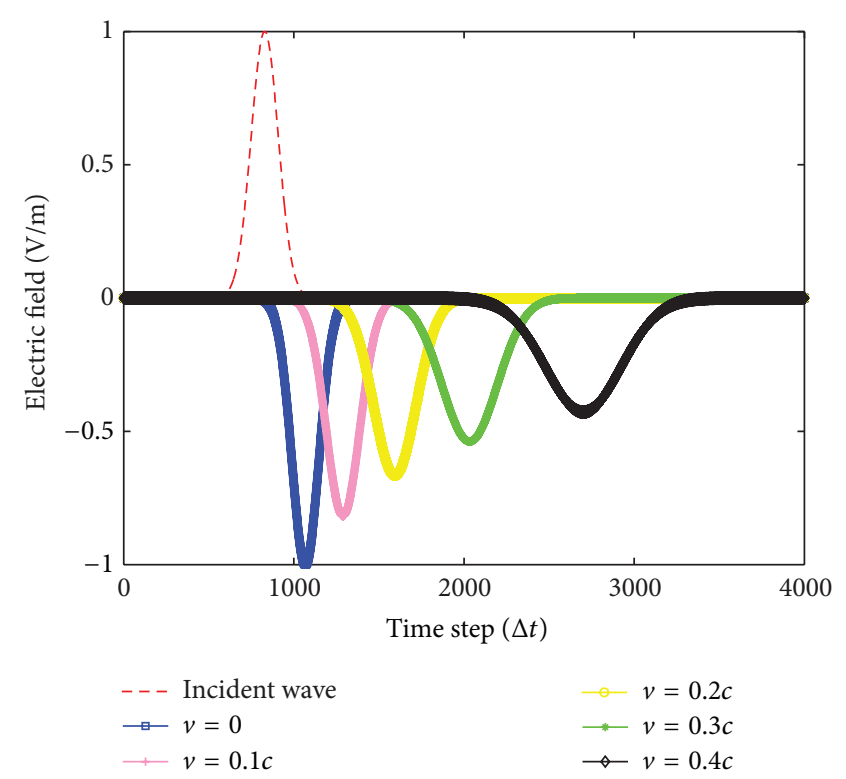

FIGURE 8: The time-domain scattered waveforms of the moving conducting surface excited by the Gaussian pulse.

Then a modulated Gaussian pulse is used to excite the moving conducting surface, which has the following form:

$$
E_{i}(n \Delta t)=-\cos (2 \pi f \cdot n \Delta t) \exp \left(-\frac{4 \pi\left(n \Delta t-t_{0}\right)^{2}}{\tau^{2}}\right),
$$

where $\tau$ and $t_{0}$ of the modulated Gaussian pulse are set to $400 \Delta t$ and $t_{0}=750 \Delta t$. The center frequency of the modulated Gaussian pulse is $1 \mathrm{GHz}$. The waveform of the modulated Gaussian pulse excitation source is shown in the Figure 9. The dashed line indicates the frequency spectrum of the excitation source in Figure 10.

Numerically simulated scattered waveforms and frequency spectrums of the conducting surface for different speeds are plotted in Figures 9 and 10, respectively. The spectrum width of the scattered field is narrowed and the peak value of the spectrum is increased with the increasing speed. It can be seen from Figure 10 that the simulated center frequencies are 1.0, 0.8180, 0.6663, 0.5379, and 0.4281, respectively, for five cases of $0,0.1 c, 0.2 c, 0.3 c$, and $0.4 c \mathrm{~m} / \mathrm{s}$.

When the conducting surface moves against the incident wave, the center frequency of the scattered field will shift to the low frequency end with an increasing moving speed. On the contrary, the center frequency will shift to the high frequency end with the increasing moving speed opposite to the propagation direction of the incident wave.

The scattered field from a two-dimensional conducting square cylinder with the size of $0.3 \mathrm{~m} \times 0.3 \mathrm{~m}$ is studied in this section. As shown in Figure 11, the incident wave is assumed to be polarized in the negative $z$-direction and propagating in the positive $x$-direction. The square cylinder is receding from the incident wave with the speed of $v$ in the positive $x$ direction. In the two-dimensional FDTD model, the spacial increment is $\Delta x=\Delta y=0.005 \mathrm{~m}$ and the time increment $\Delta t$ is 8.3333 picoseconds. A Gaussian pulse with maximum electric 


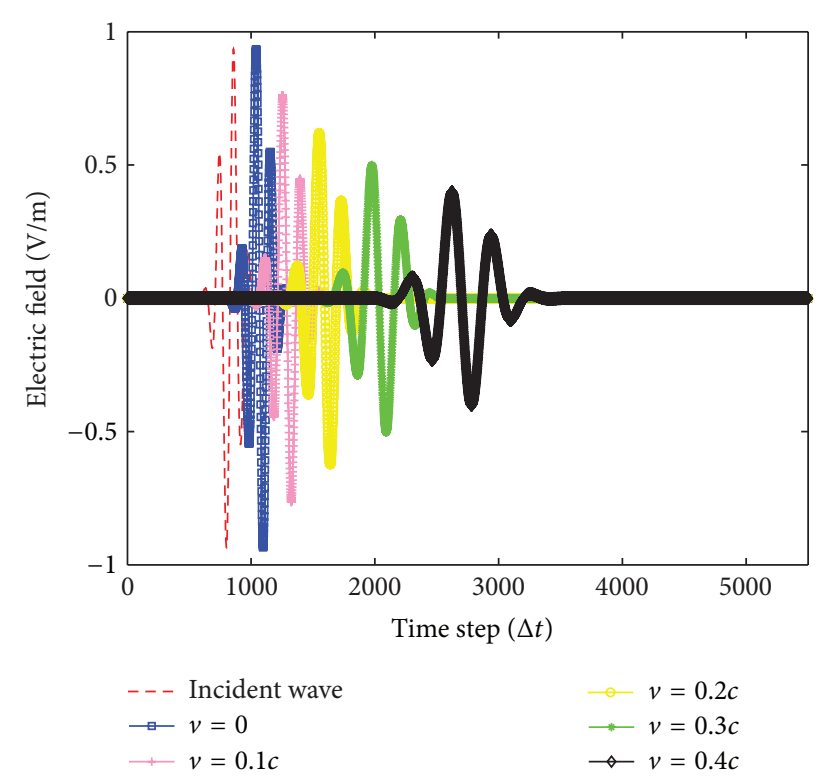

FIGURE 9: The time-domain scattered waveforms of the moving conducting surface excited by the modulated Gaussian pulse.

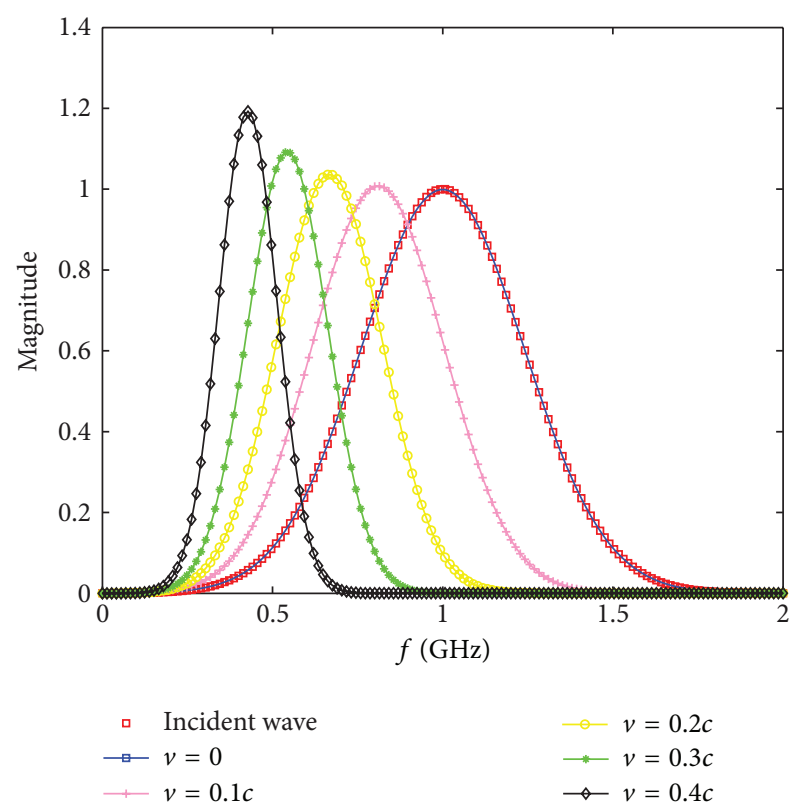

FIGURE 10: Frequency spectrums of the moving conducting surface excited by the modulated Gaussian pulse.

field amplitude of $1 \mathrm{~V} / \mathrm{m}$ is adopted to excite the moving conducting square cylinder. Referring to (11), $\tau$ and $t_{0}$ of the Gaussian pulse are set to $\tau=80 \Delta t$ and $t_{0}=50 \Delta t$, respectively. When the square cylinder is static, the horizontal distance from the observation point to the left boundary of the square cylinder is $0.075 \mathrm{~m}$ and the vertical distance is $0.15 \mathrm{~m}$.

In the simulation, the speed of the moving conducting square cylinder takes four cases of $0,0.1 c, 0.2 c$, and $0.3 \mathrm{~cm} / \mathrm{s}$. Time-domain scattered waveforms received by the observation point for the four cases are plotted in Figure 12,

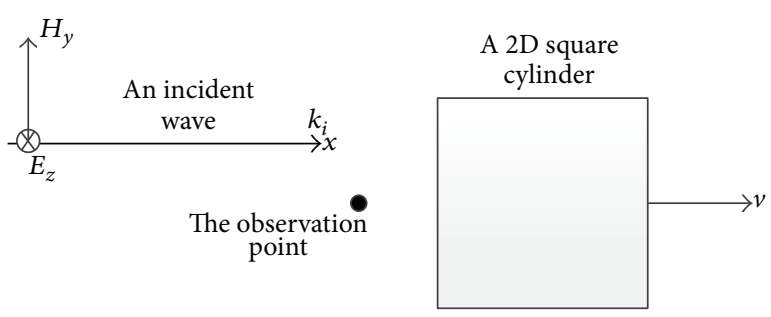

FIGURE 11: A plane wave normally incident on a moving conducting square cylinder.

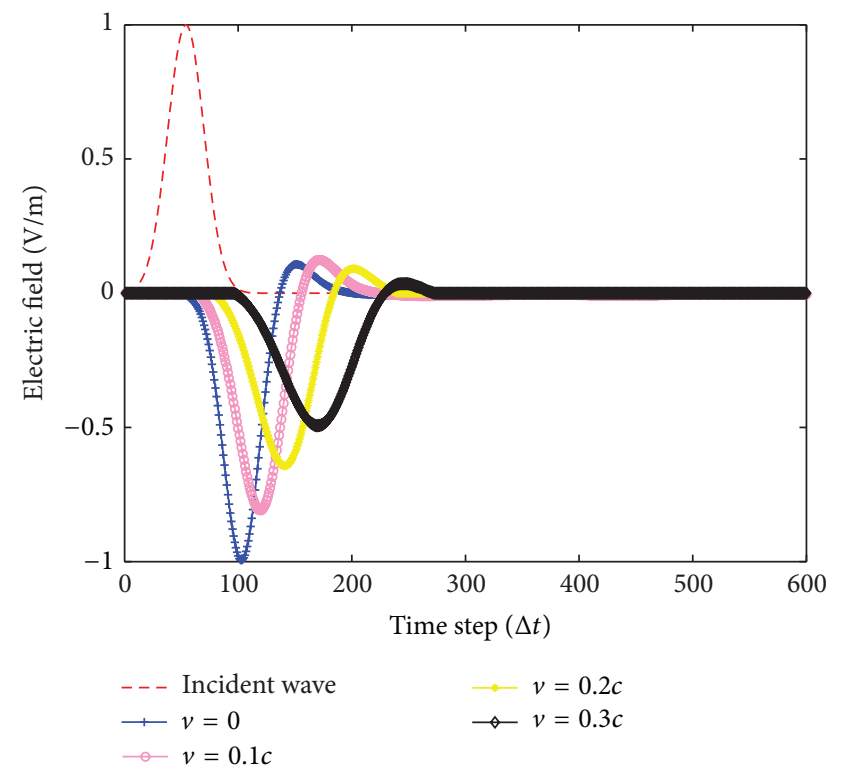

FIGURE 12: The time-domain scattered waveforms of the moving conducting square cylinder excited by the Gaussian pulse.

respectively. From Figure 12, a pulse-width of a scattered wave is broadened with the increasing moving speed. The amplitudes of simulated scattered pulses are equal to 1.0, $0.8091,0.6441$, and $0.4936 \mathrm{~V} / \mathrm{m}$, respectively. Compared with Figure 8, the amplitudes of scattered pulses from a moving square cylinder are less than those of scattered pulses from a moving infinite surface. The main reason is that the amplitude of scattered wave from a two-dimensional object declines with the spreading distance.

Subsequently, a modulated Gaussian pulse is used to excite the moving conducting square cylinder. $\tau$ and $t_{0}$ of the modulated Gaussian pulse are set to $\tau=400 \Delta t$ and $t_{0}=$ $450 \Delta t$, respectively. The center frequency of the modulated Gaussian pulse is $1 \mathrm{GHz}$. The waveform of the modulated Gaussian pulse excitation source is shown in Figure 13.

Numerically simulated scattered waveforms and frequency spectrums of the conducting square cylinder for different speeds are plotted in Figures 13 and 14, respectively. The spectrum width of the scattered field is narrowed with the increasing moving speed. Compared with Figure 10, the peak values of the spectrum of scattered waves from a moving square cylinder are less than those of the spectrum of 


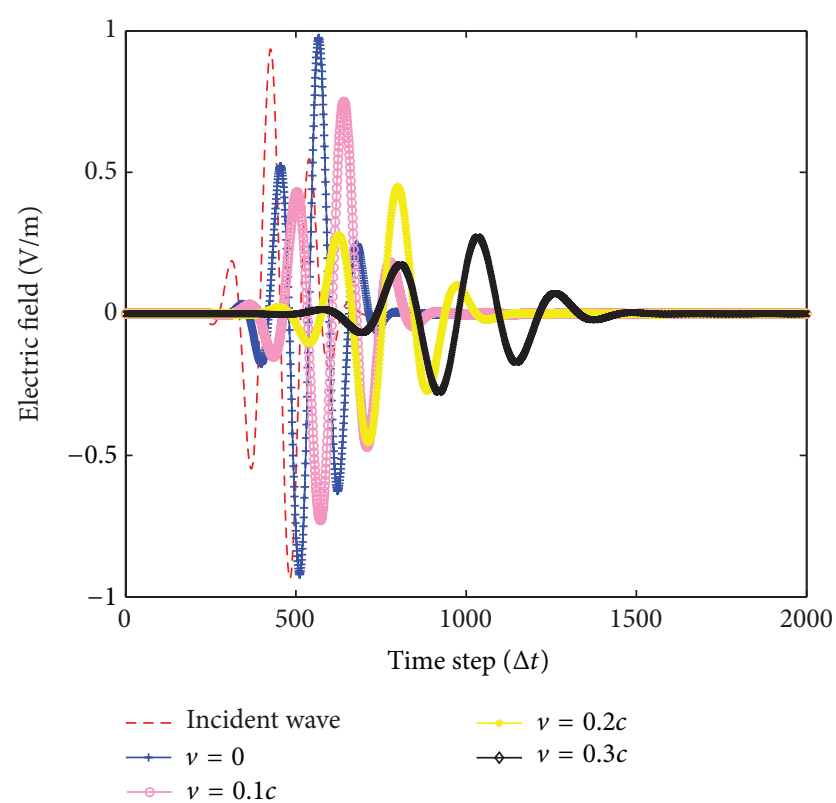

FIGURE 13: The time-domain scattered waveforms of the moving conducting square cylinder excited by the modulated Gaussian pulse.

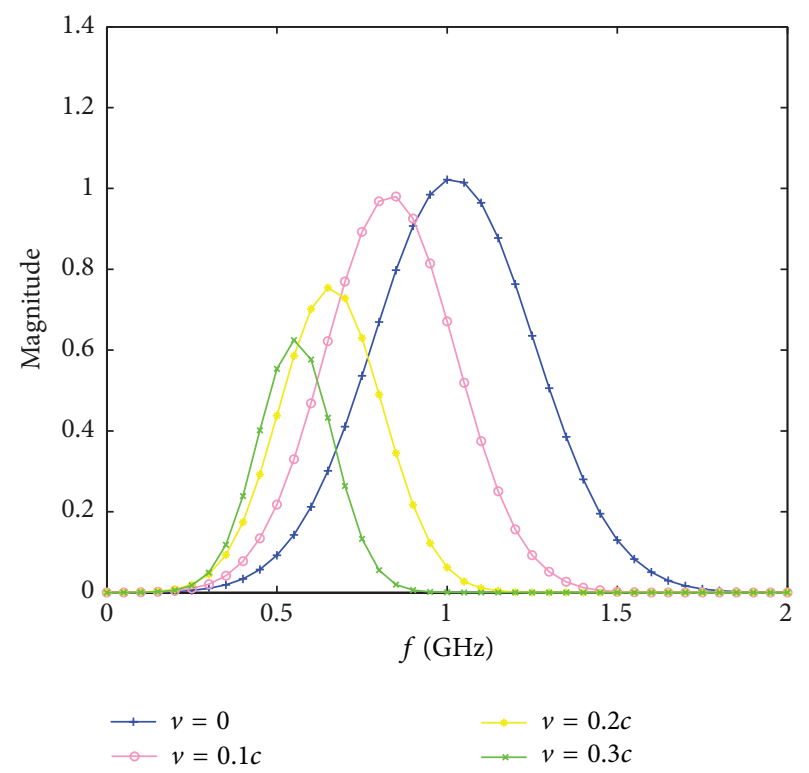

FIGURE 14: Frequency spectrums of the moving conducting square cylinder excited by the modulated Gaussian pulse.

scattered waves from a moving infinite surface. The reason is that the energy density decreases with the spreading distance.

\section{Conclusions}

Electromagnetic scattering from a moving perfectly conducting object in the steady state (one frequency) and transient state (broadband) has been analyzed by the proposed numerical method based on the FDTD algorithm. Relativistic boundary conditions are incorporated to solve the electromagnetic field on the moving boundary. And an efficient technique is introduced to calculate the electromagnetic field around the moving conducting object. The numerical results illustrate that the range of the frequency and amplitude shift of the scattered field varies with the moving speed of the object. By comparing simulated results with theoretical results, the accuracy and validity of the numerical approach are evaluated.

Contrary to other numerical methods using the Lorentz transformation, the proposed numerical approach uses no system transformation and gives the time-domain solutions directly. It is convenient to analyze broadband scattering properties of moving bodies. This approach can be applied to many practical engineering problems, such as detecting the speed and direction of moving satellites or aircrafts by radar echoes.

\section{Conflict of Interests}

The authors declare that there is no conflict of interests regarding the publication of this paper.

\section{Acknowledgments}

The research work was supported by National Natural Science Foundation of China under Grant no. 61201070, Shanghai Key Laboratory of Navigation and Location Based Services, and the Fundamental Research Funds for the Central Universities.

\section{References}

[1] W. A. Strauss, "The existence of the scattering operator for moving obstacles," Journal of Functional Analysis, vol. 31, no. 2, pp. 255-262, 1979.

[2] R. C. Costen and D. Adamson, "Three dimensional derivation of the electrodynamic jump conditions and momentum-energy laws at a moving boundary," Proceedings of the IEEE, vol. 53, no. 9, pp. 1181-1196, 1965.

[3] B. L. Michielsen, G. C. Herman, A. T. Dehoop, and D. D. Zutter, "Three-dimensional relativistic scatterings of electromagnetic waves by an object in uniform translation motion," Journal of Mathematical Physics, vol. 22, pp. 2716-2722, 1981.

[4] I. Arnaoudov and G. Venkov, "Scattering of electromagnetic plane waves by a spheroid uniformly moving in free space," Mathematical Methods in the Applied Sciences, vol. 29, no. 12, pp. 1423-1433, 2006.

[5] V. Georgiev, "Inverse scattering problem for the Maxwell equations outside moving body," Annales de l'Institut Henri Poincare, vol. 50, no. 1, pp. 37-70, 1989.

[6] M. Ho, "Simulation of scattered em fields from rotating cylinder using passing center swing back grids technique in two dimensions," Progress in Electromagnetics Research, vol. 92, pp. 79-90, 2009.

[7] K. S. Yee, "Numerical solution of initial boundary value problems involving Maxwell's equations in isotropic media," IEEE Transactions on Antennas and Propagation, vol. 14, pp. 302-307, 1966. 
[8] A. Taflove and M. E. Brodwin, "Numerical solution of steadystate electromagnetic scattering problems using the timedependent Maxwell's equations," IEEE Transactions on Microwave Theory and Techniques, vol. 23, no. 8, pp. 623-630, 1975.

[9] A. Taflove and S. C. Hagness, Computational Electrodynamics: The Finite-Difference Time-Domain Method, Artech House, Norwood, NJ, USA, 3rd edition, 2005.

[10] S. Sahrani and M. Kuroda, "Numerical analysis of the electromagnetic wave scattering from a moving dielectric body by overset grid generation method," in Proceedings of the IEEE Asia-Pacific Conference on Applied Electromagnetics (APACE '12), pp. 11-13, Melaka Malaysia, December 2012.

[11] K. S. Zheng, J. Z. Li, G. Wei, and J. D. Xu, "Analysis of Doppler effect of moving conducting surfaces with Lorentz-FDTD method," Journal of Electromagnetic Waves and Applications, vol. 27, no. 2, pp. 149-159, 2013.

[12] S. Kharkovsky, J. T. Case, M. T. Ghasr, R. Zoughi, S. W. Bae, and A. Belarbi, "Application of microwave 3D SAR imaging technique for evaluation of corrosion in steel rebars embedded in cement-based structures," AIP Conference Proceedings, vol. 1430, pp. 1516-1523, 2012.

[13] F. Harfoush, A. Taflove, and G. A. Kriegsmann, "Numerical technique for analyzing electromagnetic wave scattering from moving surfaces in one and two dimensions," IEEE Transactions on Antennas and Propagation, vol. 37, no. 1, pp. 55-63, 1989.

[14] J. A. Kong, Electromagnetic Wave Theory, Wiley, New York, NY, USA, 2nd edition, 1990.

[15] J. De Moerloose and D. De Zutter, "Surface integral representation radiation boundary condition for the FDTD method," IEEE Transactions on Antennas and Propagation, vol. 41, no. 7, pp. 890-896, 1993.

[16] J. Cooper, "Scattering of electromagnetic fields by a moving boundary: the one-dimensional case," IEEE Transactions on Antennas and Propagation, vol. AP-28, no. 6, pp. 791-795, 1980.

[17] F. Kural, F. Arikan, O. Arikan, and M. Efe, "Performance evaluation of track association and maintenance for a MFPAR with doppler velocity measurements," Progress in Electromagnetics Research, vol. 108, pp. 249-275, 2010. 

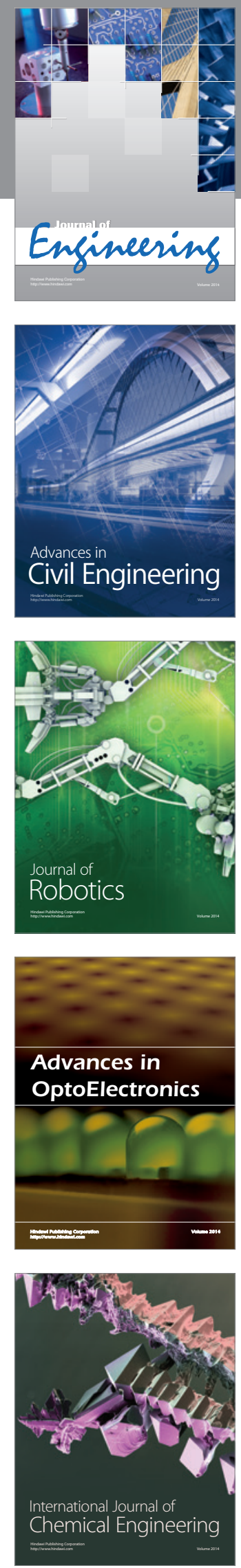

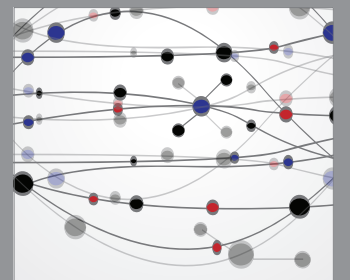

The Scientific World Journal
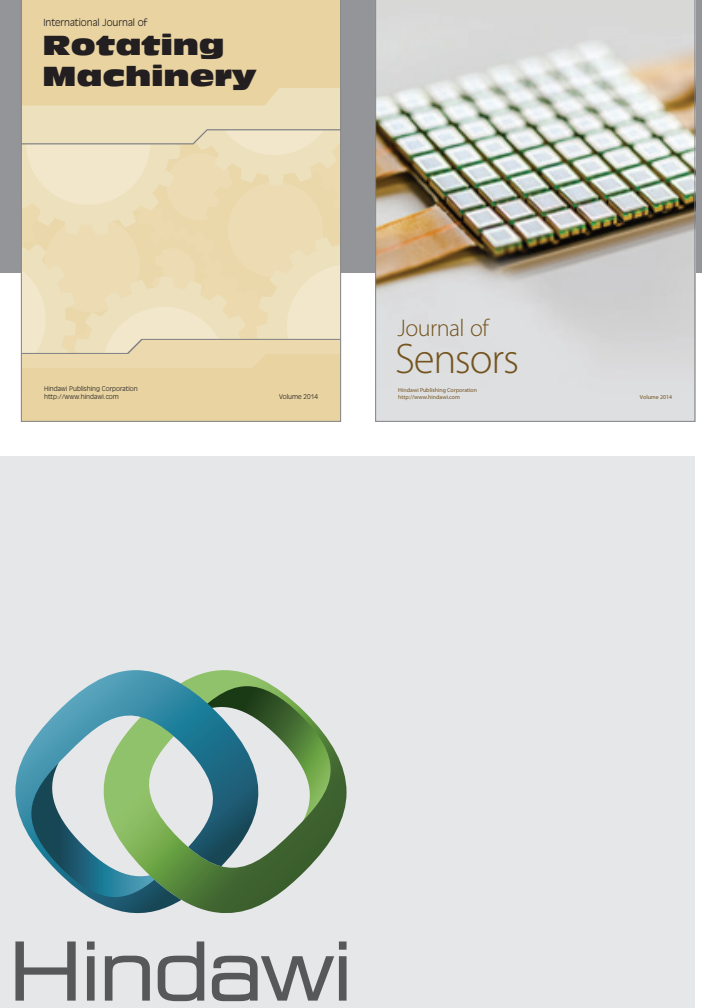

Submit your manuscripts at http://www.hindawi.com
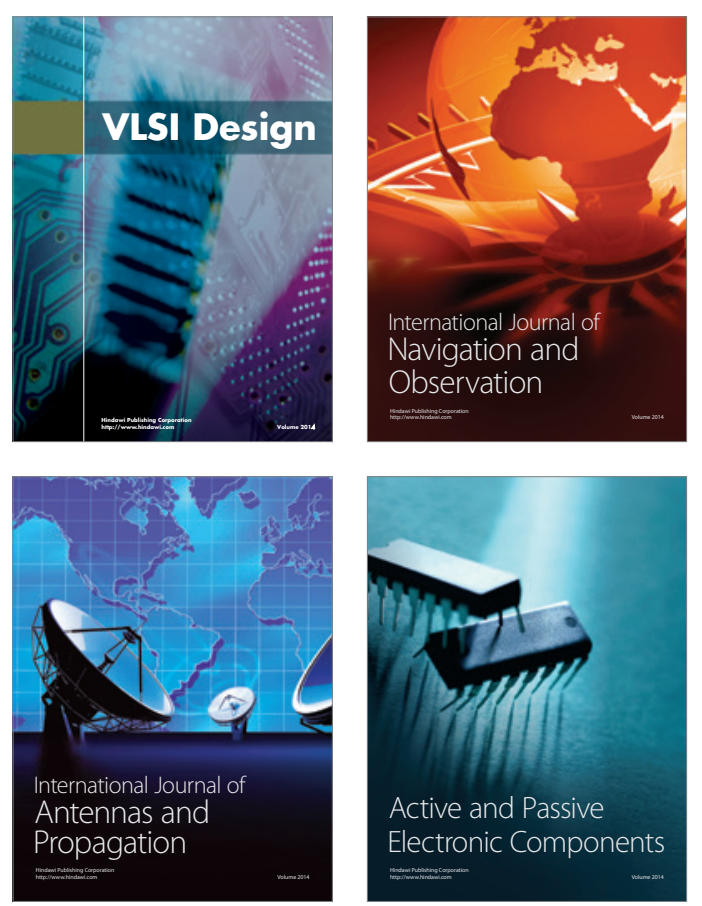
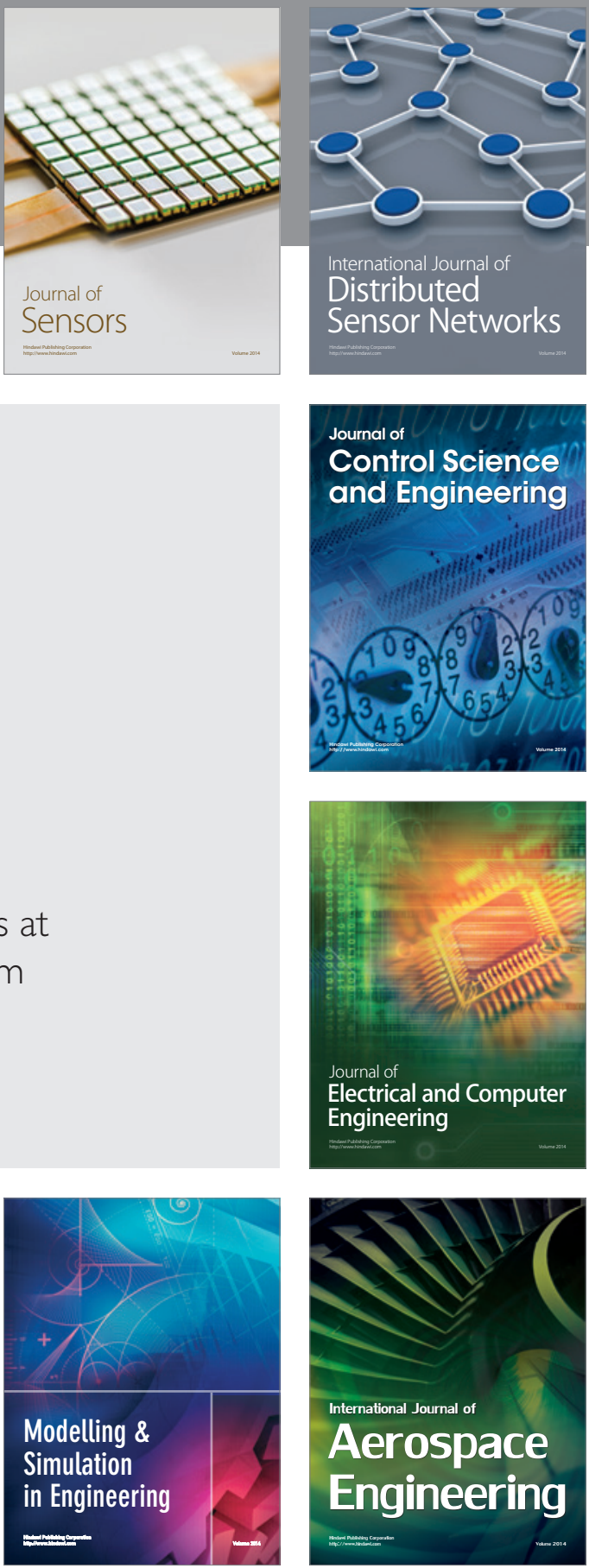

Journal of

Control Science

and Engineering
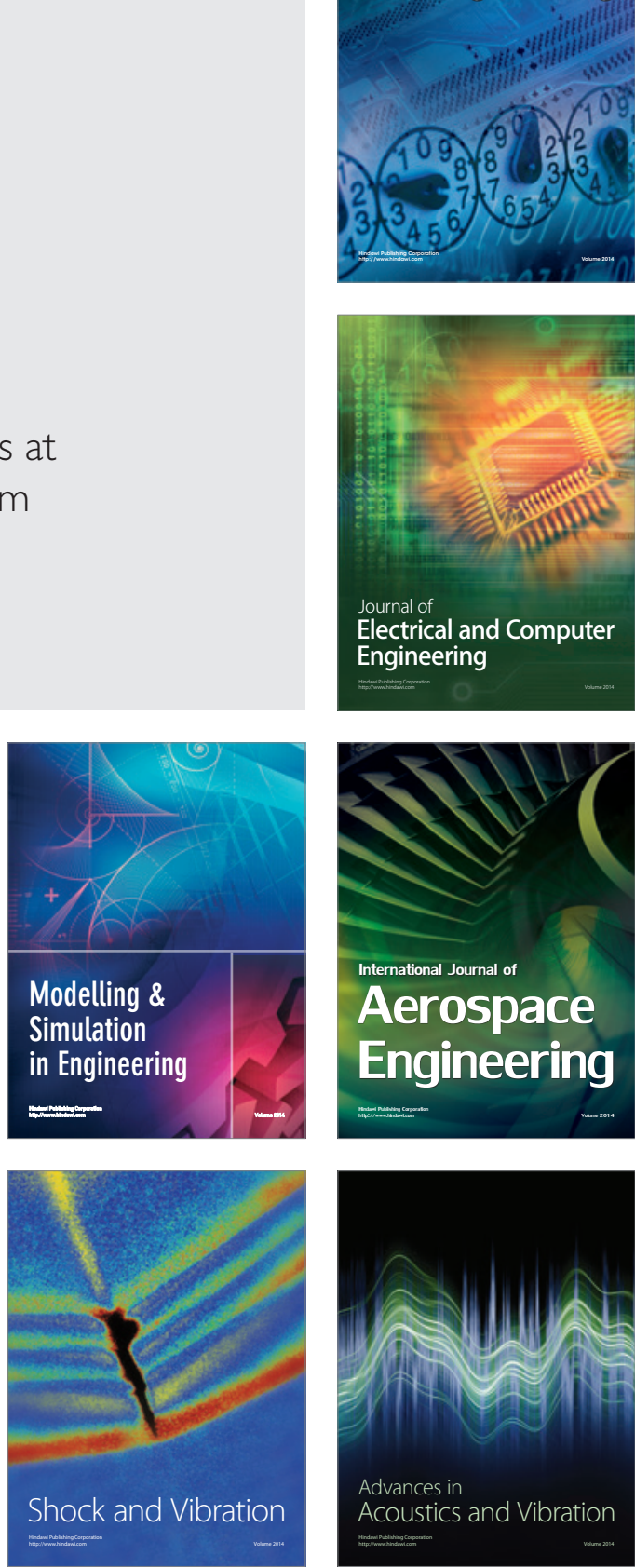